\title{
Contribution of transmission in HIV-positive men who have sex with men to evolving epidemics of sexually transmitted infections in England: an analysis using multiple data sources, 2009-2013
}

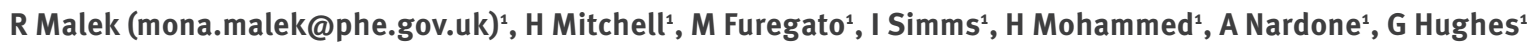

1. Public Health England, London, United Kingdom

Citation style for this article:

Malek R, Mitchell H, Furegato M, Simms I, Mohammed H, Nardone A, Hughes G. Contribution of transmission in HIV-positive men who have sex with men to evolving epidemics of sexually transmitted infections in England: an analysis using multiple data sources, 2009-2013. Euro Surveill. 2015;20(15):pii=21093. Available online: http://www.eurosurveillance.org/ViewArticle.aspx?Articleld=21093

HIV seroadaptive behaviours may have contributed to greater sexually transmitted infection (STI) transmission in HIV-positive men who have sex with men (MSM) and to the global increase in STIs. Using multiple national surveillance data sources and population survey data, we estimated the risk of STIs in HIV-positive MSM and assessed whether transmission in HIV-positive MSM has contributed to recent STI epidemics in England. Since 2009, an increasing proportion of STIs has been diagnosed in HIV-positive MSM, and currently, the population rate of acute bacterial STIs is up to four times that of HIV-negative or undiagnosed MSM. Almost one in five of all diagnosed HIV-positive MSM in England had an acute STI diagnosed in 2013. From 2009 to 2013, the odds of being diagnosed with syphilis increased from 2.71 (95\% confidence interval (Cl) 2.41-3.05, p<0.001) to 4.05 (95\% $\mathrm{Cl} 3.70-4.45$, pro.001) in HIV-positive relative to HIVnegative/undiagnosed MSM. Similar trends were seen for gonorrhoea and chlamydia. Bacterial STI re-infection rates were considerably higher in HIV-positive MSM over a five-year follow-up period, indicative of rapid transmission in more dense sexual networks. These findings strongly suggest that the sexual health of HIV-positive MSM in England is worsening, which merits augmented public health interventions and continued monitoring.

\section{Introduction}

The United Kingdom (UK) has seen a steady increase in diagnoses of sexually transmitted infections (STIS) in the last decade, in particular, in men who have sex with men (MSM). From 2012 to 2013, gonorrhoea and syphilis diagnoses in MSM increased by $26 \%$ and $12 \%$ respectively [1]. The emergence of resistance and reduced sensitivity to frontline treatments of gonorrhoea is of global concern and may have contributed to high levels of gonorrhoea transmission in MSM [2-4]. Additionally, outbreaks of less common STIs such as Lymphogranuloma venereum (LGV) [5], together with shigellosis (which can be sexually transmissible) [6] in this population are of particular concern.

There is increasing evidence that HIV-positive MSM in the UK are disproportionately affected by STIs. Recent data demonstrated that of MSM diagnosed with syphilis, $35 \%$ were HIV positive [7]. HIV-positive MSM have also been found to account for more than $80 \%$ of LGV cases [5]. In part, these observations may reflect seroadaptive behaviours in HIV-positive MSM [8], in which different sexual practices such as unprotected (i.e. condomless) anal intercourse are adopted according to the reported HIV status of both partners in order to reduce the risk of transmitting or acquiring HIV. Seroadaptive behaviours vary considerably and include serosorting (limiting sexual partners to those with the same HIV status as themselves), strategic positioning (adopting a specific sexual position according to the HIV status of one's partner), withdrawal before ejaculation, and negotiating around viral load [9]. However, seroadaptive behaviours may come at a cost of increased transmission of STIs [10-12].

The UK has a growing population of HIV-positive MSM [13] and recent studies suggest that they may be increasingly adopting seroadaptive behaviours $[8,14]$. In this study we used multiple surveillance data sources and population survey data to estimate the risk of STIs in HIV-positive MSM and assessed whether transmission in HIV-positive MSM has contributed to recent STI epidemics in England.

\section{Methods}

\section{Sources of data}

Descriptive and comparative data analyses of the incidence of STIs were undertaken using data from GUMCADv2 (Genitourinary Medicine Clinic Activity Dataset) [15]. The Survey of Prevalent HIV Infections Diagnosed (SOPHID) [16], the third National Survey 
of Sexual Attitudes and Lifestyles (Natsal-3) [17] and census data from the Office for National Statistics (ONS) [18] were employed to estimate denominator populations.

GUMCADv2 is a mandatory electronic pseudoanonymised (i.e. contains the sex, age and hospital/ clinic number of each patient but no patient identifiable information such as name, date of birth or postcode of residence is included) [19] patient-level dataset submitted to Public Health England (PHE) by all genitourinary medicine (GUM) clinics in England. The dataset contains information on all STI diagnoses and services provided for each patient as well as information on patient demographic such as sexual orientation, age, sex, ethnicity, area of residence and country of birth [19]. A unique patient identifier is assigned to each patient attending a given GUM clinic, allowing subsequent visits by the same patient to the same clinic to be identified.

SOPHID is a cross-sectional survey of all persons with diagnosed HIV infection who attend for HIV care at an NHS site in England, Wales and Northern Ireland. Age, sex, probable route of HIV infection, ethnicity, antiretroviral therapy (ART) status, $C_{4}$ cell count, region of residence and region of care provider are measured for each calendar year.

Natsal- 3 is a nationally representative survey conducted between 2010 and 2012 on 15,162 individuals and provides information on key sexual behaviours, risk factors and also includes biological sampling and testing.

ONS is the national statistical institute for the UK and is responsible for collecting and publishing statistics related to the economy, population and society at national, regional and local levels.

\section{Study population}

MSM were defined as men who reported a homosexual or bisexual orientation at least once over the study period. MSM were defined as diagnosed HIV positive (hereafter referred to as 'HIV-positive' MSM) if they were diagnosed with HIV at least six weeks before their STI diagnosis, as newly diagnosed with HIV if they were diagnosed within six weeks of their STI diagnosis, and as HIV-negative/undiagnosed if there was no evidence of an HIV diagnosis in their GUMCADv2 record. For the analysis of acute STIs, STI population rates and association between STI outcomes and HIV status, MSM with new HIV diagnoses were grouped with those of negative or unknown status and referred to collectively as 'HIV-negative/undiagnosed', as the newly diagnosed men were assumed to be undiagnosed at the time of their STI exposure.

\section{Data analysis}

Acute sexually transmitted infections in known HIV-positive men who have sex with men Episodes of acute STI diagnoses including gonorrhoea (acute and complicated), syphilis (primary, secondary and early latent), chlamydia, genital warts (first episode) and genital herpes (first episode) and HIV status in MSM were identified using Sexual Health and HIV Activity Property Type (SHHAPT) codes from GUMCADV2 for the years 2008 to 2013. Acute gonorrhoea includes all new cases of uncomplicated gonorrhoea of the lower genitourinary tract, anorectum, mouth, throat and adult conjunctivitis; complicated gonorrhoea includes all upper genitourinary tract complications (such as pelvic inflammatory disease and epididymitis) and systemic complications [20]. The proportion of STI diagnoses which were in HIV-positive MSM was calculated for each STI by year.

Sexually transmitted infection population rates in HIV-negative/undiagnosed and HIV-positive men who have sex with men

The rates of acute bacterial (gonorrhoea, chlamydia and syphilis) and acute viral STIs (first episode of genital warts and first episode of genital herpes) in HIV-positive and HIV negative/undiagnosed MSM were compared from 2009 to 2013. The numerators were individual HIV-positive and negative/undiagnosed MSM presenting at GUM clinics each year using data from GUMCADv2. The denominator for HIV-positive MSM was identified from SOPHID. The proportion of men aged 15 to 74 years who are MSM was estimated using data from Natsal-3. An estimated $2.6 \%$ of men in the UK had at least one male sexual partner in the past five years across all age groups of men [21]. This was applied to the mid-year population estimates from the ONS for the number of men aged 15 to 74 years for each year [22,23], and the estimated number of HIV-positive MSM was subtracted from this to calculate the denominator for HIV-negative/undiagnosed MSM. The results of a sensitivity analysis (data not shown) showed a small effect when using the upper and lower limits of the confidence interval (2.1\% and $3 \%$ respectively) of the estimation of men in the UK having at least one male sexual partner in the past five years across all ages of men. For the most recent year of analysis, the range for bacterial STIs in HIV-negative MSM was 33 per 1,000 to 48 per 1,000 .

\section{Associations between sexually transmitted infection outcomes and HIV status}

Univariate and multivariate logistic regression models were prepared for the MSM population attending GUM clinics in England for each individual MSM diagnosed with an acute bacterial STI (gonorrhoea, chlamydia and syphilis) for each year from 2009 to 2013 inclusive. Explanatory variables included all demographic variables from GUMCADv2 (age, ethnicity, continent of birth, and area of residence), diagnosed HIV status, and number of tests in the previous 12-month period 
Proportion of acute sexually transmitted infection diagnoses in men who have sex with men which were in HIV-positive men who have sex with men, England, 2009-2013

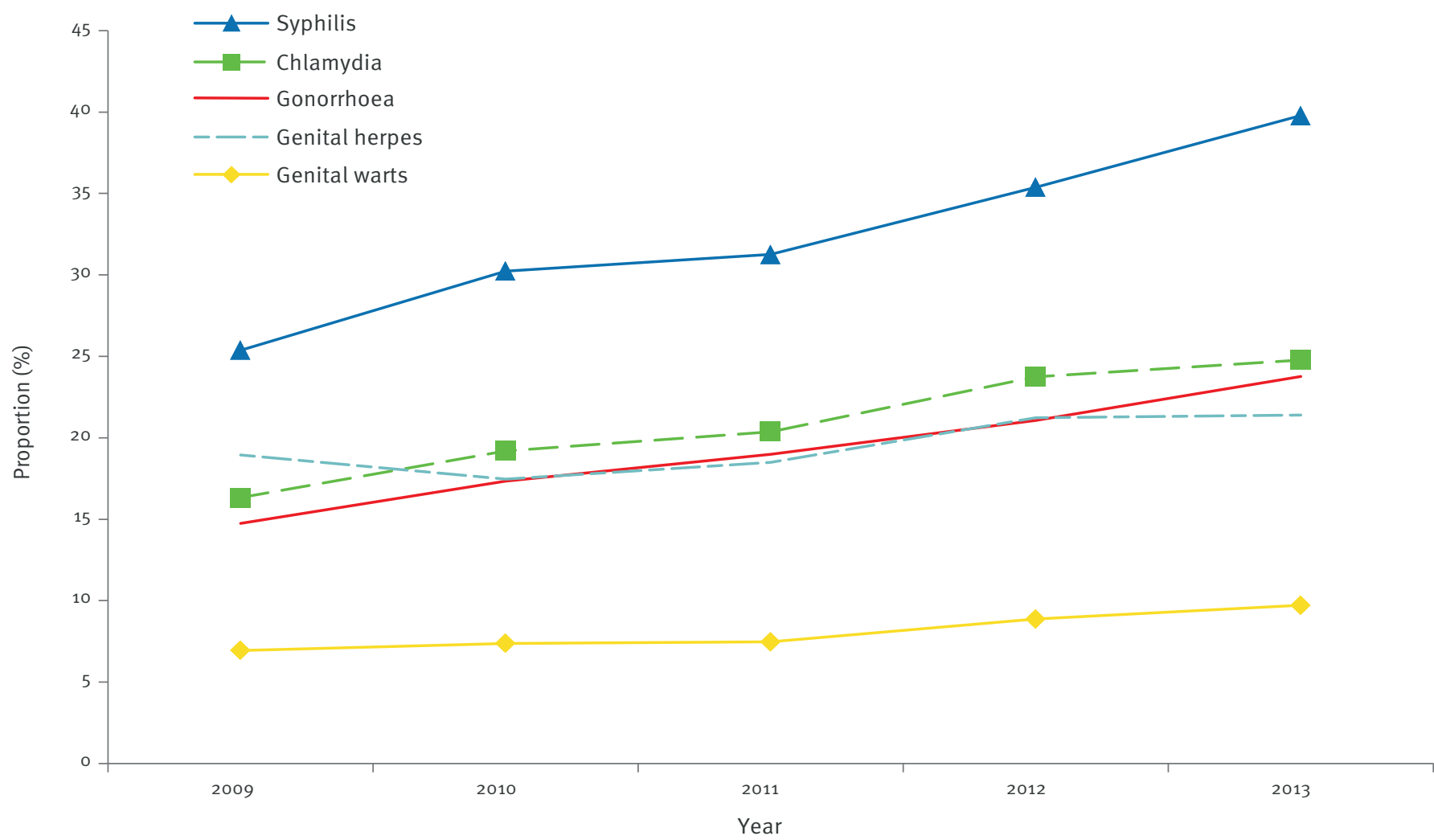

for each respective bacterial STI; all were included in the final multivariate model.

\section{Gonorrhoea and chlamydia re-infection rates} by HIV status

The probability of patients who became re-infected with the most common bacterial STIs (gonorrhoea and chlamydia) within one year was estimated by the Kaplan-Meier method. Patients became at-risk from 42 days after the time of first attendance with gonorrhoea and chlamydia [24] and were censored at the end of the study period (31 December 2013). Data for calculating re-infection rates was obtained from GUMCADv2.

All statistical analyses were undertaken using Stata version 12 (StataCorp, College Station, Texas, US). $P$ values $<0.05$ were considered to be statistically significant.

\section{Results}

During the study period, the number of MSM attending GUM clinics recorded in GUMCADv2 increased from 78,226 in 2009 to 117,410 in 2013. The total number of MSM attendances increased from 241,676 to 316,250. The number of MSM in England estimated using Natsal-3 and ONS was 501,895 in 2009, increasing to 516,416 in 2013.
Acute sexually transmitted infections in known HIVpositive men who have sex with men

The proportion of acute STI diagnoses in MSM that were in HIV-positive MSM is shown in Figure 1. Overall, from 2009 to 2013, this proportion increased for all acute STIs: from $25 \%$ to $40 \%$ for syphilis, $16 \%$ to $25 \%$ for chlamydia, $15 \%$ to $24 \%$ for gonorrhoea, $19 \%$ to $21 \%$ for genital herpes and $7 \%$ to $10 \%$ for genital warts.

Sexually transmitted infection population rates in HIV-negative/undiagnosed and HIV-positive men who have sex with men

The rate of acute bacterial STIS in HIV-positive MSM nearly trebled (64 per 1,000 to 161 per 1,000), and increased from 3.2 times higher than the rate in HIVnegative/undiagnosed MSM in 2009 to 4.2 times higher in 2013 (Figure 2). For HIV-negative/undiagnosed MSM, the rate of acute bacterial STIs also increased to a lesser degree (19 per 1,000 to 38 per 1,000). The rate of acute viral STIs in HIV-positive MSM was approximately twice that observed in HIV-negative/undiagnosed MSM and remained fairly stable over the five year period ( 15 to 18 per 1,000 in HIV-positive and 8 to 9 per 1,000 in negative/undiagnosed MSM). This indicates that the rate of acute STIs in HIV-positive MSM was close to one in five (179 per 1,000). 
Rates of acute bacterial and acute viral sexually transmitted infections in HIV-positive and negative/undiagnosed men who have sex with men per 1,000 men who have sex with men, England, 2009-2013

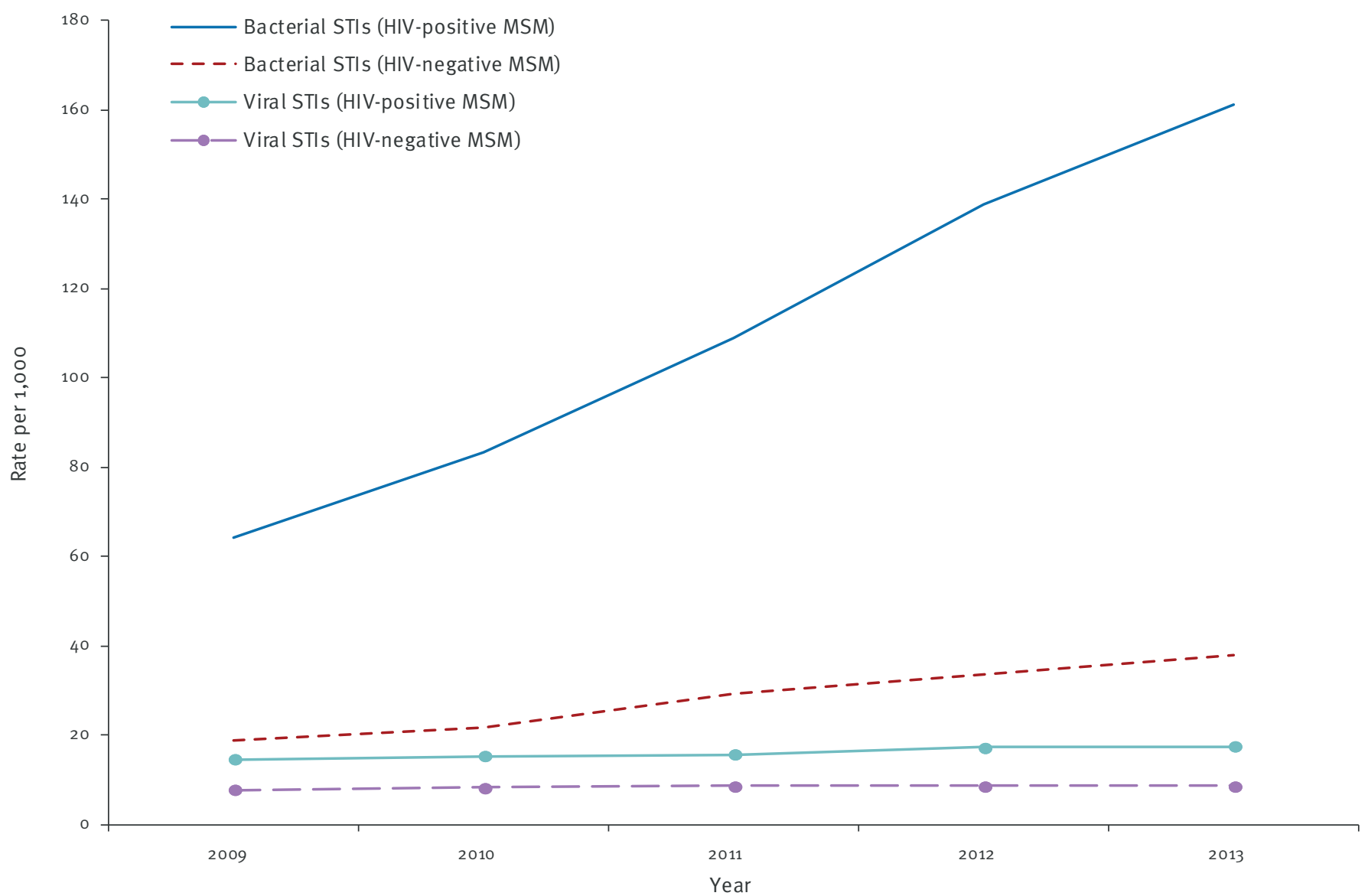

MSM: men who have sex with men; STI: sexually transmitted infection.

Bacterial STIs: gonorrhoea, chlamydia and syphilis

Viral STIs: first episode of genital warts and first episode of genital herpes

\section{Associations between sexually transmitted} infection outcomes and HIV status

Table 1 shows the final multivariate logistic regression models for gonorrhoea, chlamydia and syphilis adjusted for all explanatory variables. Data are presented for the most recent available year only. Table 2 shows the change in the adjusted odds ratio over time by HIV status. When compared with HIV-negative/undiagnosed MSM, the adjusted odds ratio (aOR) of being diagnosed with gonorrhoea, chlamydia and syphilis was significantly higher in HIV-positive MSM in all years analysed and increased over time ( $p<0.001)$.

\section{Gonorrhoea and chlamydia re-infection rates by HIV status}

The estimated probability of gonorrhoea and chlamydia re-infection is shown in Figure 3. A total of 34,090 and 31,206 MSM diagnosed with gonorrhoea and chlamydia respectively were included. The probability of repeat infection with gonorrhoea was estimated at $36.6 \%$ in HIV-positive, $33.2 \%$ in newly diagnosed and $22.7 \%$ in HIV-negative/undiagnosed MSM at the end of the 5 year follow-up period. For chlamydia, the estimated probability of repeat infection was $31.6 \%$ in HIV-positive, $23.7 \%$ in newly diagnosed and $17.3 \%$ in HIV-negative/undiagnosed MSM over the same period.

\section{Discussion}

For the first time, we have estimated the relative contribution of HIV-positive MSM to STI transmission over time in England. Since 2009, an increasing proportion of STIs has been diagnosed in HIV-positive MSM, and currently, the population rate of acute bacterial STIs is up to four times that of HIV-negative or undiagnosed MSM. We estimate that almost one in five of all diagnosed HIV-positive MSM in England had an acute STI diagnosed in 2013. The odds of being diagnosed with gonorrhoea, syphilis and chlamydia were significantly higher in HIV-positive relative to HIV-negative/undiagnosed MSM and increased over time. Re-infection rates of bacterial STIs were also considerably higher in HIV-positive MSM over a five-year follow-up period, indicative of rapid transmission in more dense sexual networks. 


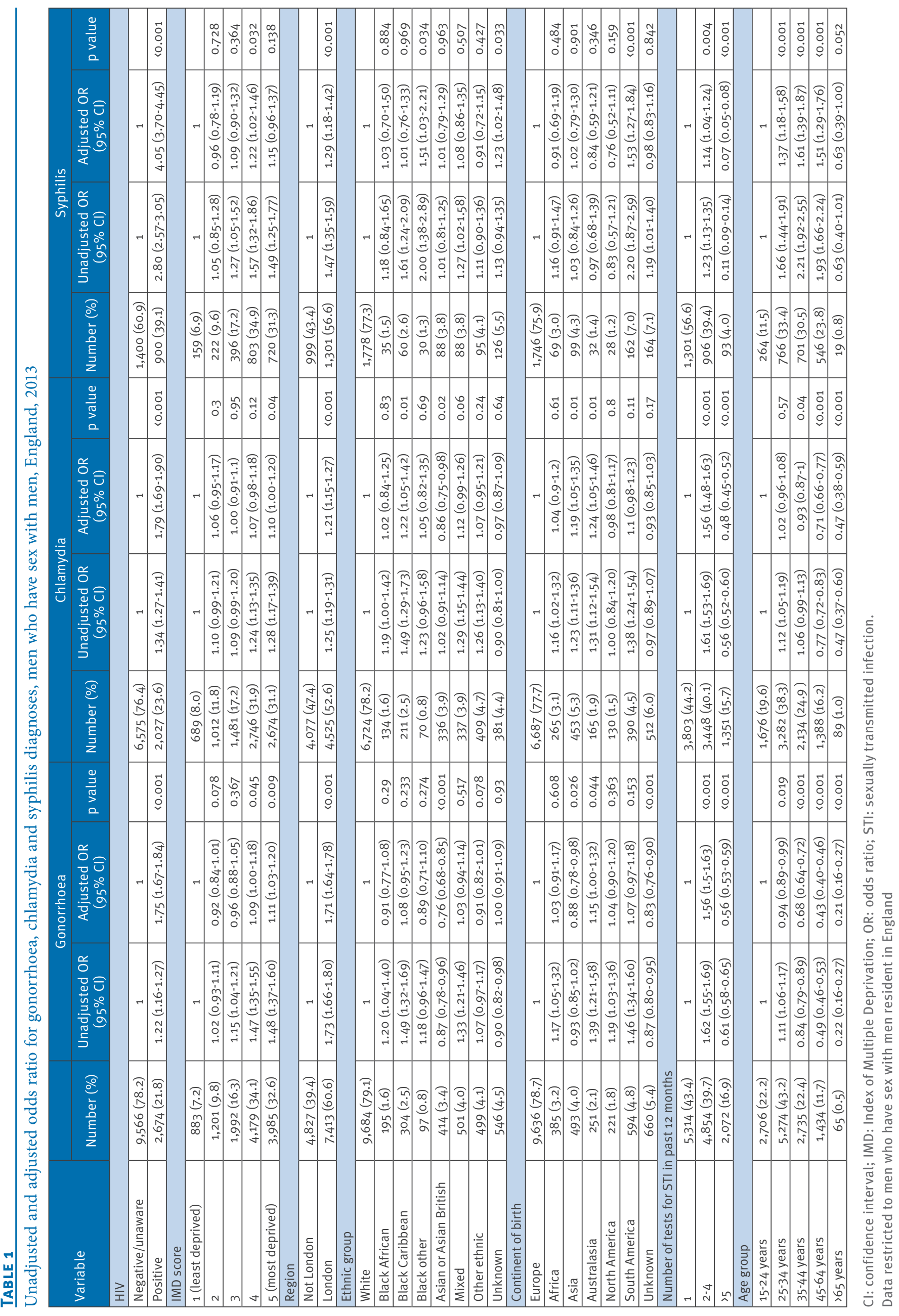




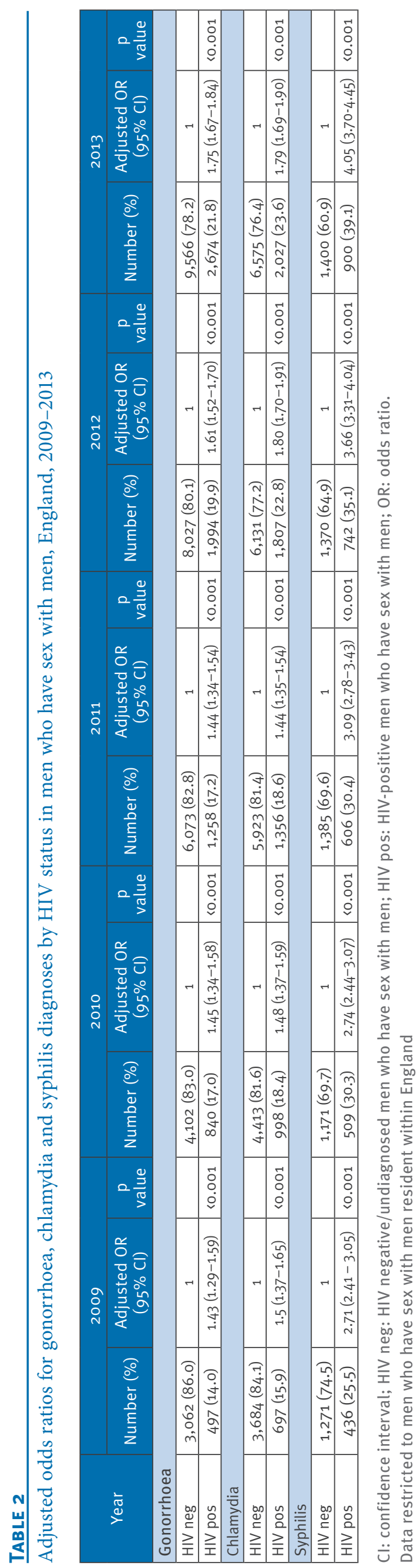

These findings strongly suggest that the sexual health of HIV-positive MSM in England is worsening. They are consistent with data from a cross-sectional survey of men in commercial gay venues in London, Brighton, Manchester, Glasgow and Edinburgh which demonstrated that numbers of STIs diagnosed in the previous 12 months were higher in MSM known to be HIV-infected compared with uninfected men (aOR 7.2, 95\% Cl 4.63-11.17) [25], and similar studies in Europe $[26,27]$. A study of LGV re-infection in the UK also found that, at baseline, repeaters were more likely to be HIVpositive compared with non-repeaters [28].

It seems probable that these changes in STI transmission patterns in HIV-positive MSM reflect increasing adoption of HIV seroadaptive behaviours and their impact on sexual network structures. HIV-positive MSM reporting seroadaptive behaviours are at higher risk of STIs compared with HIV-negative MSM [10-12]. One study reported that HIV-positive MSM practising seroadaptive behaviours had a threefold increased risk of bacterial STIs, with almost a third of HIV-positive MSM reporting an STI in the past year, compared with $9 \%$ of HIV-negative MSM [11]. However, despite the considerable changes in STI transmission patterns in HIVpositive MSM seen in our study, the degree to which transmission in HIV-positive MSM engaging in seroadaptive behaviours is fuelling current STI epidemics in England is unclear. The recent emergence of relatively rare infections such as LGV and S. flexneri in the UK has been strongly and predominantly associated with transmission in HIV-positive MSM $[5,6,29]$. Likewise for syphilis, our study showed that $39.1 \%$ of cases in MSM in England were known to be HIV-positive, and this is consistent with data from the United States, Australia and Europe $[26,27,30,31]$. The duration of infectiousness with Shigella, LGV and syphilis may be short as these infections are typically symptomatic, so it is highly likely that their transmission is being sustained in highly active sexual networks of HIV-positive MSM engaging in seroadaptive behaviours [32]. However, fewer than $25 \%$ of MSM diagnosed with chlamydia and gonorrhoea (and even less with viral STIs) in our study were HIV-positive. Therefore, while seroadaptive behaviours in HIV-positive MSM may be making an important contribution to the transmission of chlamydia, gonorrhoea and viral STIs in MSM, they are not necessary to sustain infection at endemic levels in the wider MSM population.

HIV seroadaptive behaviours will likely have other negative or unintended consequences for the sexual health of MSM. The presence of an STI may compromise the health of HIV-positive MSM through several mechanisms including a reduction in $\mathrm{CD}_{4}$ cell count as well as acute increases in HIV viral load, which may compromise effective antiretroviral therapy [33-35]. In addition, STIs may also increase HIV infectiousness by facilitating HIV shedding in the genital tract or rectal mucosa [36]. Further, evidence suggests that a low viral load may reduce the probability of infecting 


\section{FIGURE 3}

Estimated probability of repeat gonococcal infection (A) and of repeat chlamydial infection (B) in men who have sex with men attending genitourinary medicine clinics by HIV co-infection, England, 2009-2013

A
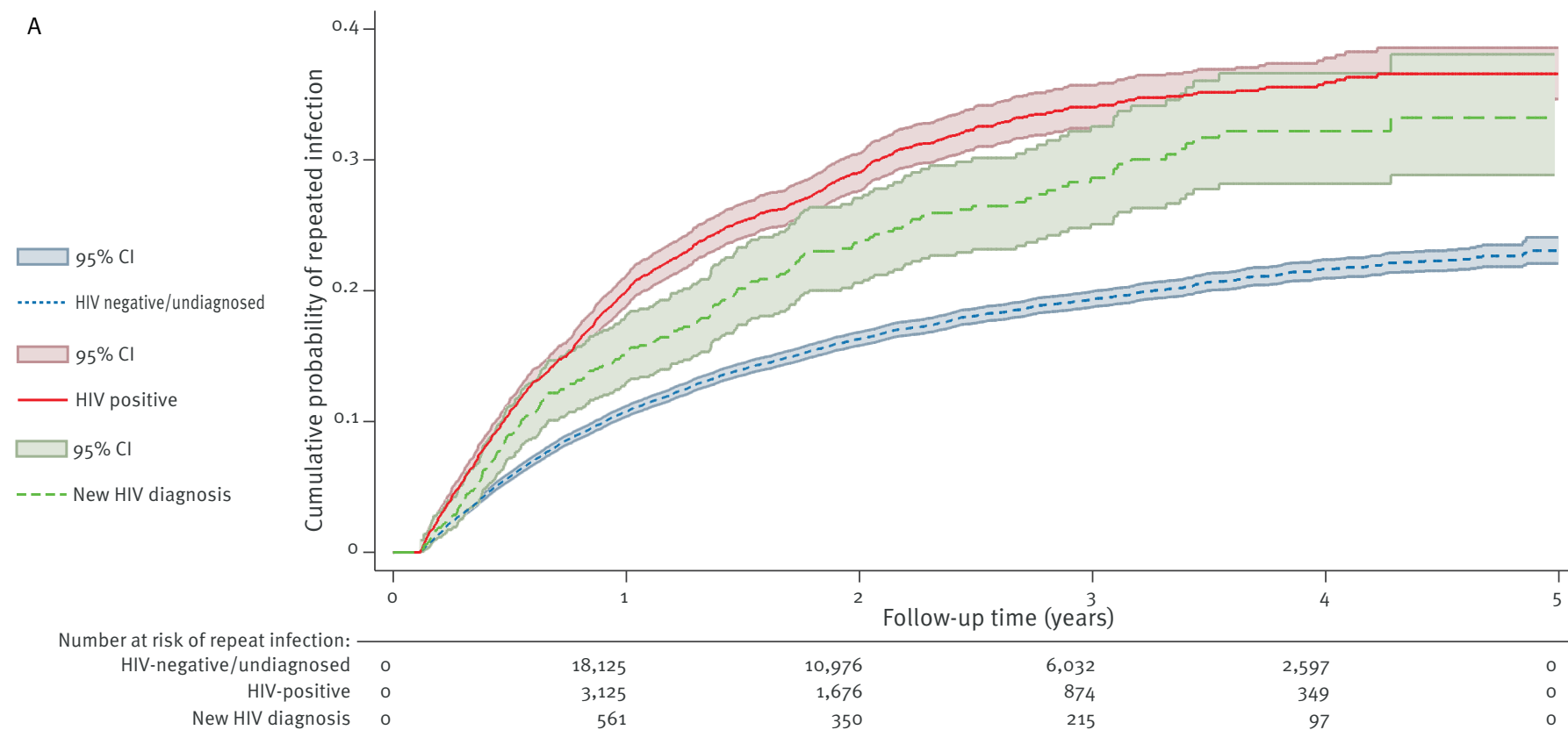

B

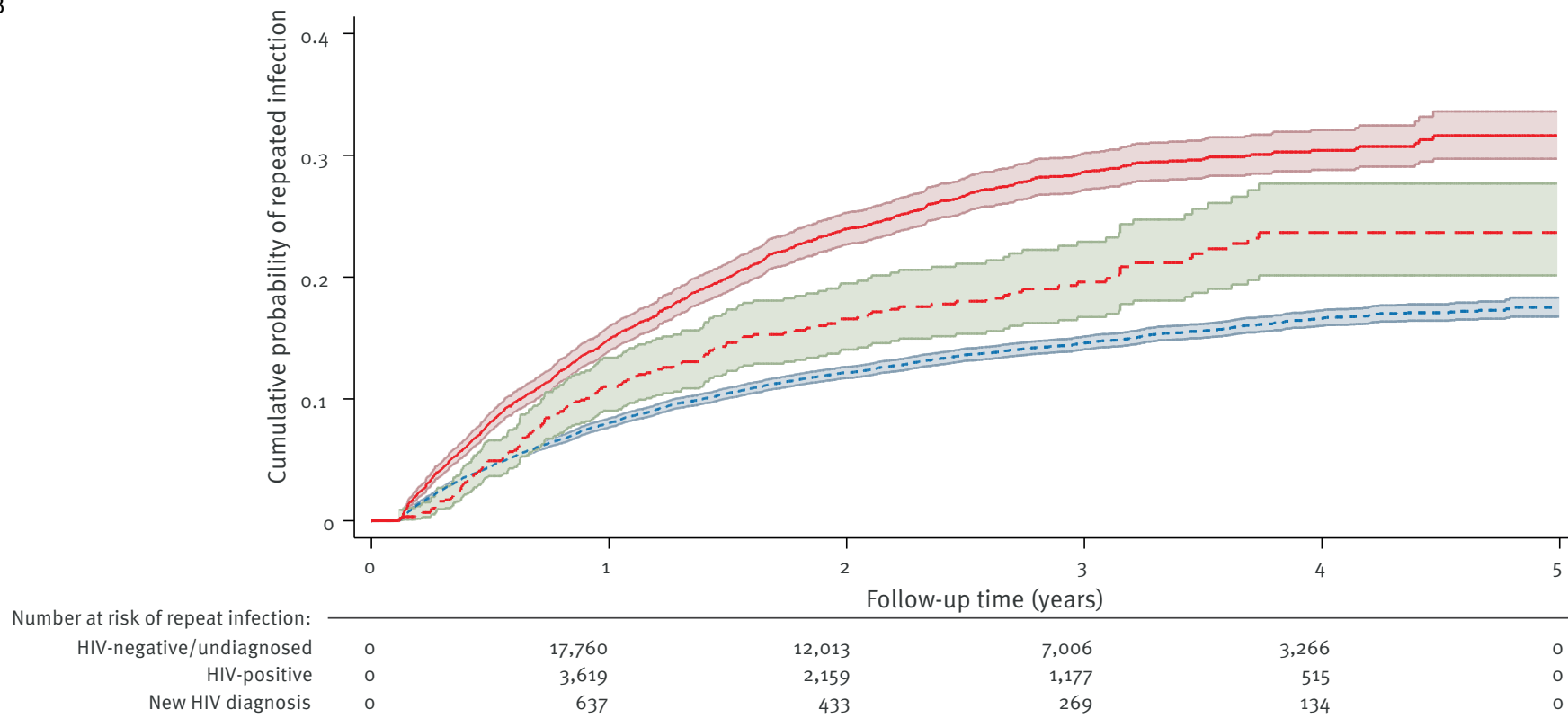

a sexual partner [37-39], thus, HIV-positive MSM may engage in seroadaptive behaviours when they receive highly active antiretroviral therapy (HAART) or have an undetectable viral load. However, surveys of gay commercial venues and gyms in London, Glasgow and Edinburgh suggest the proportion of MSM reporting unprotected anal intercourse with partners of unknown or discordant HIV status has increased, leading to risk of HIV as well as other STI transmission $[8,14]$.

A study by Fox et al. showed significant reduction in self-reported HIV transmission-risk behaviour in MSM recently diagnosed with HIV, with patients reporting greater condom use and fewer sexual partners [40].
However, these may have limited impact on STI incidence due to the various routes of transmission of STIs (such as oral, digital and use of sex toys). Furthermore, in MSM recently diagnosed with HIV, those reporting continued transmission-risk behaviour were more likely to have another STI [40]. Thus, health promotion activities should also consider the broader context around sexual risk-taking in MSM, especially in those diagnosed with HIV. The recent Public Health England framework for promoting the health and well being of MSM highlighted the interaction of mental health, alcohol and drug use, and sexual risk behaviour [41]. There is increasing concern on the interaction between drug use and STIs, especially in HIV-positive 
MSM, and that in developing appropriate interventions and services, the specific needs of HIV-positive MSM should be considered [29]. Furthermore, the high incidence of asymptomatic STIs, especially in extra-genital sites $[42,43]$ emphasises the need to promote regular screening for STIs in HIV-positive individuals $[44,45]$. Nonetheless, surveys of healthcare providers in the US have reported significant barriers, especially in screening for gonorrhoea and chlamydia, which include time constraints, difficulty obtaining a sexual history, language and cultural barriers, and patient confidentiality concerns [46].

There are several limitations of this study. There may be a degree of ascertainment bias in the assessment of HIV status in MSM in patients with a longer history in GUMCADv2. However, sensitivity analysis (data not shown) was performed by identifying HIV-positive MSM from a retrospective review of a single year of GUMCADv2 data, and there was only a minimal impact on the results. The probability of repeat infection may also have been underestimated as, in this analysis, repeat diagnosis was used as a proxy measure for repeat infection. Some patients will have become re-infected but will remain undiagnosed, as only patients who returned to the same clinic for testing were assessed in the analysis. This is a limitation of GUMCADv2, as it allows only longitudinal patient data within a particular clinic or service and attendances by the same patient at different clinics cannot be monitored [19]. However, the data quality and completeness of GUMCADv2 is extremely high with $100 \%$ submissions from GUM clinics [19]. HIV-positive MSM engaged in care are also more likely to return regularly for STI screening during clinic visits and this may have contributed to the proportion and rates of STIs observed. Diagnosis of STIs (including HIV) is dependent on screening practices and frequency of screening. It is therefore not a true measure of incidence of infection but provides a good proxy for infection. A further limitation is the estimation of the true size of the MSM population in the UK. However, the methodology employed in Natsal-3 is among the most robust to estimate the size of this population. Furthermore, between Natsal-2 and Natsal-3, there was no significant increase in the proportion of men reporting same sex partners in the past 5 years [21]. Thus, the results of this longitudinal data analysis provide valuable insights into the complexity and evolution of STI epidemics in England.

The presence of an increasing proportion and rates of acute STIs in HIV-positive MSM, a population which also has higher rates of repeat infection and reports higher risk sexual behaviour, presents an increased risk for the sexual health of all MSM. This therefore merits public health action through improved monitoring and intervention. Currently, the collection of behavioural data is being piloted alongside that of clinical and socio-demographic data and this will allow further insights into the impact of seroadaptive practices to be explored in the future. Improved public health interventions with a holistic approach focussing on promoting condom use, reducing high risk behaviour and increasing the frequency of STI testing in MSM should be a priority.

\section{Conflict of interest}

None declared.

\section{Authors' contributions}

Gwenda Hughes and Anthony Nardone devised the study, advised on data analysis and participated in interpreting the data. Ramona Malek, Holly Mitchell and Martina Furegato carried out statistical analysis, participated in data analysis and intepreting the data. Ian Simms and Hamish Mohammed participated in interpreting the data. Ramona Malek prepared the first draft of the manuscript. All authors contributed to the final manuscript.

\section{References}

1. Health Protection Report: weekly report. Sexually Transmitted Infections and Chlamydia screening in England, 2014; 8(24), Public Health England. Available from: https://www.gov.uk/ government/uploads/system/uploads/attachment_data/ file/326935/hpr2414.pdf

2. Ison CA, Town K, Obi C, Chisholm S, Hughes G, Livermore DM, et al.; GRASP collaborative group. Decreased susceptibility to cephalosporins in gonococci: data from the Gonococcal Resistance to Antimicrobials Surveillance Programme (GRASP) in England and Wales, 2007-2011. Lancet Infect Dis. 2013;13(9):762-8. http://dx.doi.org/10.1016/S14733099(13)70143-9 PMID:23764300

3. Cole MJ, Spiteri G, Chisholm SA, Hoffmann S, Ison CA, Unemo $M$, et al. Emerging cephalosporin and multidrug-resistant gonorrhoea in Europe. Euro Surveill. 2014;19(45):20955. http://dx.doi.org/10.2807/1560-7917.ES2014.19.45.20955. Available from: http://www.eurosurveillance.org/ViewArticle. aspx?Articleld=20955 PMID:25411689

4. Centers for Disease Control and Prevention (CDC). Cephalosporin susceptibility among Neisseria gonorrhoeae isolates--United States, 2000-2010. MMWR Morb Mortal Wkly Rep. 2011;60(26):873-7. PMID:21734634

5. Hughes G, Alexander S, Simms I, Conti S, Ward H, Powers C, et al.; LGV Incident Group. Lymphogranuloma venereum diagnoses among men who have sex with men in the U.K.: interpreting a cross-sectional study using an epidemic phase-specific framework. Sex Transm Infect. 2013;89(7):542 7. http://dx.doi.org/10.1136/sextrans-2013-051051 PMID:23851189

6. Borg ML, Modi A, Tostmann A, Gobin M, Cartwright J, Quigley $C$, et al. Ongoing outbreak of Shigella flexneri serotype $3 a$ in men who have sex with men in England and Wales, data from 2009-2011. Euro Surveill. 2012;17(13):20137. Available from: http://www.eurosurveillance.org/ViewArticle. aspx?Articleld=20137 PMID:22490381

7. Sexually transmitted infections in men who have sex with men in the UK: 2011 report. Health Protection Agency. November 2011.

8. Lattimore S, Thornton A, Delpech V, Elford J. Changing patterns of sexual risk behavior among London gay men: 1998-2008. Sex Transm Dis. 2011;38(3):221-9. http://dx.doi.org/10.1097/ OLQ.obo13e3181f2ebe1 PMID:20921930

9. Kennedy CE, Bernard LJ, Muessig KE, Konda KA, Akl EA, Lo Y-R et al. Serosorting and HIV/STI infection among HIV-negative MSM and transgender people: a systematic review and meta-analysis to inform WHO guidelines. Journal of Sexually Transmitted Diseases. Volume 2013: Article ID 583627, 8 pages.

10. Marcus U, Schmidt AJ, Hamouda O. HIV serosorting among HIV-positive men who have sex with men is associated with increased self-reported incidence of bacterial sexually transmissible infections. Sex Health. 2011;8(2):184-93. http:// dx.doi.org/10.1071/SH10053 PMID:21592432

11. Mayer KH, O'Cleirigh C, Skeer M, Covahey C, Leidolf E, Vanderwarker R, et al. Which HIV-infected men who have sex with men in care are engaging in risky sex and acquiring 
sexually transmitted infections: findings from a Boston community health centre. Sex Transm Infect. 2010;86(1):66-70. http://dx.doi.org/10.1136/sti.2009.036608 PMID:19720603

12. Golden MR, Stekler J, Hughes JP, Wood RW. HIV serosorting in men who have sex with men: is it safe? I Acquir Immune Defic Syndr. 2008;49(2):212-8. http://dx.doi.org/10.1097/ QAl.ob013e31818455e8 PMID:18769346

13. Public Health England. National HIV Surveillance data tables. No.1:2013 HIV \& AIDS New Diagnosis and Deaths database. The Survey of Prevalent Infections Diagnosed (SOPHID). Updated 6 October 2014. [Accessed 21 Jan 2015]. Available from: https:// www.gov.uk/government/statistics/hiv-data-tables

14. Wallace LA, Li J, McDaid LM. HIV prevalence and undiagnosed infection among a community sample of gay and bisexual men in Scotland, 2005-2011: implications for HIV testing policy and prevention. PLoS ONE. 2014;9(3):e90805. Available from: http://dx.doi.org/10.1371/journal.pone.0090805 PMID:24621479

15. Health Protection Agency. GUMCADv2 Guidance to Clinic Staff. 2013:1-36. Available from: https://www.gov.uk/ genitourinary-medicine-clinic-activity-dataset-gumcadv2

16. Public Health England. SOPHID metadata, 2nd version. 20 Sep 2013. Available from: https://www.gov.uk/ hiv-surveillance-systems

17. Erens B, Phelps A, Clifton S, Mercer C, Tanton C, Hussey D, et al. Methodology of the third British National Survey of Sexual Attitudes and Lifestyles (Natsal-3). Sex Transm Infect. 2014;90(2):84-9.

18. Office for National Statistics. United Kingdom. [Accessed 12 Jan 2015]. Available from: http://www.ons.gov.uk/ons/index. html

19. Savage El, Mohammed H, Leong G, Duffell S, Hughes G. Improving surveillance of sexually transmitted infections using mandatory electronic clinical reporting: the genitourinary medicine clinic activity dataset, England, 2009 to 2013. Euro Surveill. 2014;19(48):20981. http://dx.doi.org/10.2807/15607917.ES2014.19.48.20981. PMID:25496573

20. NHS data dictionary. [Internet]. Available from: http://www. datadictionary.nhs.uk/version2/data dictionary/messages/ central_returns/community/kc60/kc60_1_fr.asp

21. Mercer CH, Tanton C, Prah P, Erens B, Sonnenberg P, Clifton $S$, et al. Changes in sexual attitudes and lifestyles in Britain through the life course and over time: findings from the National Surveys of Sexual Attitudes and Lifestyles (Natsal) Lancet. 2013;382(9907):1781-94. Available from: http://dx.doi. org/10.1016/S0140-6736(13)62035-8 PMID:24286784

22. Office for National Statistics. Mid-1971 to mid-2012 population estimates: quinary age groups for constituent countries in the UK: estimated resident population. United Kingdom. Available from: www.ons.gov.uk/ons/rel/pop-estimate/ population-estimates-for-uk--england-and-wales--scotlandand-northern-ireland/mid-2001-to-mid-2010-revised/rft---mid2001-to-mid-2010-population-estimates-analysis-tool.zip

23. Office for National Statistics. Mid-2013 population estimates analysis tool. 25 June 2014. United Kingdom. [Accessed 12 Jan 2015]. Available from: www.ons.gov.uk/ons/rel/pop-estimate/ population-estimates-for-uk--england-and-wales--scotlandand-northern-ireland/2013/rft---mid-2013-populationestimates-analysis-tool.zip

24. Hughes G, Nichols T, Peters L, Bell G, Leong G, Kinghorn G. Repeat infection with gonorrhoea in Sheffield, UK: predictable and preventable? Sex Transm Infect. 2013;89(1):38-44. http:// dx.doi.org/10.1136/sextrans-2012-050495 PMID:22717472

25. Williamson LM, Dodds JP, Mercey DE, Hart GJ, Johnson AM. Sexual risk behaviour and knowledge of HIV status among community samples of gay men in the UK. AIDS. 2008;22(9):1063-70. http://dx.doi.org/10.1097/ QAD.ob013e3282f8afgb PMID:18520350

26. Dougan S, Evans BG, Elford J. Sexually transmitted infections in Western Europe among HIV-positive men who have sex with men. Sex Transm Dis. 2007;34(10):783-90. PMID:17495592

27. European Centre for Disease Prevention and Control (ECDC). STI and HIV prevention in men who have sex with men in Europe. Stockholm: ECDC; 2013. Available from: http://ecdc. europa.eu/en/publications/Publications/STI-HIV-preventionMSM-in-Europe-21-Feb-2013.pdf

28. Rönn M, Hughes G, White P, Simms I, Ison C, Ward H. Characteristics of LGV repeaters: analysis of LGV surveillance data. Sex Transm Infect. 2014;90(4):275-8. http://dx.doi. org/10.1136/sextrans-2013-051386 PMID:24431182

29. Gilbart VL, Simms I, Gobin M, Oliver I, Hughes G. Highrisk drug practices in men who have sex with men. Lancet. 2013;381(9875):1358-9. http://dx.doi.org/10.1016/S01406736(13)60882-X PMID:23601946
30. Centres for Disease Control and Prevention. STDs in men who have sex with men. 2011 Sexually Transmitted Disease Surveillance. [Accessed 12 Jan 2015]. Available from: http:// www.cdc.gov/std/stats $11 / \mathrm{msm}$.htm

31. Jin F, Prestage GP, Zablotska I, Rawstorne P, Kippax SC, Donovan $\mathrm{B}$, et al. High rates of sexually transmitted infections in HIV positive homosexual men: data from two community based cohorts. Sex Transm Infect. 2007;83(5):397-9. http:// dx.doi.org/10.1136/sti.2007.025684 PMID:17556503

32. Rönn MM, Ward H. The association between lymphogranuloma venereum and HIV among men who have sex with men: systematic review and meta-analysis. BMC Infect Dis. 2011;11(1):70. http://dx.doi.org/10.1186/1471-2334-11-70 PMID:21418569

33. Ward H, Rönn M. Contribution of sexually transmitted infections to the sexual transmission of HIV. Curr Opin HIV AIDS. 2010;5(4):305-10. http://dx.doi.org/10.1097/ COH.obo13e32833a8844 PMID:20543605

34. Palacios R, Jiménez-Oñate F, Aguilar $M$, Galindo MJ, Rivas $P$, Ocampo A, et al. Impact of syphilis infection on HIV viral load and CD4 cell counts in HIV-infected patients. J Acquir Immune Defic Syndr. 2007;44(3):356-9. http://dx.doi.org/10.1097/ QAI.ob013e31802ea4C6 PMID:17159654

35. Buchacz K, Patel P, Taylor M, Kerndt PR, Byers RH, Holmberg SD, et al. Syphilis increases HIV viral load and decreases CD4 cell counts in HIV-infected patients with new syphilis infections. AIDS. 2004;18(15):2075-9. http://dx.doi. org/10.1097/00002030-200410210-00012 PMID:15577629

36. Kofoed K, Gerstoft J, Mathiesen LR, Benfield T. Syphilis and human immunodeficiency virus (HIV)-1 coinfection: influence on CD4 T-cell count, HIV-1 viral load, and treatment response. Sex Transm Dis. 2006;33(3):143-8. http://dx.doi. org/10.1097/01.olq.0000187262.56820.CO PMID:16505739

37. van Kesteren NMC, Hospers HJ, Kok G. Sexual risk behavior among HIV-positive men who have sex with men: a literature review. Patient Educ Couns. 2007;65(1):5-20. http://dx.doi. org/10.1016/j.pec.2006.09.003 PMID:17098392

38. Crepaz N, Hart TA, Marks G. Highly active antiretroviral therapy and sexual risk behavior: a meta-analytic review. JAMA. 2004;292(2):224-36. http://dx.doi.org/10.1001/jama.292.2.224 PMID:15249572

39. Crepaz N, Marks G, Liau A, Mullins MM, Aupont LW, Marshall KJ, et al.; HIV/AIDS Prevention Research Synthesis (PRS) Team. Prevalence of unprotected anal intercourse among HIV-diagnosed MSM in the United States: a meta-analysis. AIDS. 2009;23(13):1617-29. http://dx.doi.org/10.1097/ QAD.obo13e32832effae PMID:19584704

40. Fox J, White PJ, Macdonald N, Weber J, McClure M, Fidler S, et al. Reductions in HIV transmission risk behaviour following diagnosis of primary HIV infection: a cohort of high-risk men who have sex with men. HIV Med. 2009;10(7):432-8. http:// dx.doi.org/10.1111/j.1468-1293.2009.00708.x PMID:19459996

41. Public Health England. Promoting the health and wellbeing of gay, bisexual and other men who have sex with men: initial findings. June 2014. [Accessed January 2015]. Available from: https://www.gov.uk/government/uploads/system/uploads/ attachment_data/file/339041/MSM_Initial_Findings__ GW2014194.pdf

42. Pérez-Hernández I, Palacios R, González-Doménech C, García V, Márquez M, Clavijo E, et al. Should screening for Chlamydia trachomatis and Neisseria gonorrhoeae in HIV-men who have sex with men be recommended? J Int AIDS Soc. 2014;17(4 Suppl 3):19661. eCollection 2014. doi: 10.7448/IAS.17.4.19661. PMID:25397411

43. Patton ME, Kidd S, Llata E, Stenger M, Braxton J, Asbel L, et al. Extragenital gonorrhea and chlamydia testing and infection among men who have sex with men--STD Surveillance Network, United States, 2010-2012. Clin Infect Dis. 2014;58(11):1564-70. http://dx.doi.org/10.1093/cid/ciu184 PMID:24647015

44. British H. IV Association (BHIVA). BHIVA Standards of Care for People Living with HIV. London; British HIV Association: 2012. Available from: http://www.bhiva.org/documents/Standardsof-care/BHIVAStandardsA4.pdf

45. Centers for Disease Control and Prevention, Health Resources and Services Administration, National Institutes of Health, American Academy of HIV Medicine, Association of Nurses in AIDS Care, International Association of Providers of AIDS Care, the National Minority AIDS Council, and Urban Coalition for HIV/AIDS Prevention Services. Recommendations for HIV Prevention with Adults and Adolescents with HIV in the United States, 2014. 2014. http://stacks.cdc.gov/view/cdc/26062

46. Carter JW Jr, Hart-Cooper GD, Butler MO, Workowski KA, Hoover KW. Provider barriers prevent recommended sexually transmitted disease screening of HIV-infected men who have sex with men. Sex Transm Dis. 2014;41(2):137-42. http:// dx.doi.org/10.1097/OLQ.0000000000000067 PMID:24413496 